\title{
Desempenho do PSF no Sul e no Nordeste do Brasil: avaliação institucional e epidemiológica da Atenção Básica à Saúde
}

\author{
Performance of the PSF in the Brazilian South \\ and Northeast: institutional and epidemiological \\ Assessment of Primary Health Care
}

Luiz Augusto Facchini 1

Roberto Xavier Piccini 1

Elaine Tomasi 2,3

Elaine Thumé 1

Denise Silva Silveira 1,3

Fernando Vinholes Siqueira 1,2

Maria Aparecida Rodrigues 1,3

\footnotetext{
1 Departamento de Medicina Social, Faculdade de Medicina, Universidade Federal de Pelotas.

Av. Duque de Caxias 250 96030-002 Pelotas RS lfachini@terra.com.br

2 Universidade Católica de Pelotas.

3 Secretaria Municipal da Saúde de Pelotas.
}

Abstract The research developed in the context of the baseline studies of the PROESF analyzed the performance of the PSF in 41 municipalities of the States Alagoas, Paraíba, Pernambuco, Piauí, Rio Grande do Norte, Rio Grande do Sul and Santa Catarina. This article describes a transversal study using an external group for comparison (traditional primary care). Forty-one Presidents of $\mathrm{Mu}$ nicipal Health Councils, 20 Municipal Health Secretaries and 32 Primary Care Coordinators were interviewed. The study characterized the structure and work process of 234 Basic Care Units, involving 4.794 workers, 4.079 children, 3.945 women, 4.060 adults and 4.006 aged people. Quality control reached 6\% of the sampled domiciles. The coverage provided by PSF between 1999 and 2004 increased more in the Northeast than in the South. Less than half of the workers were admitted through open public selection and the work under contract was more frequent in the PSF than in the traditional UBS. These findings suggest a performance of primary health care still very distant from the prescriptions of the Unified Health System. Less than half of the potential users frequented the UBS in their area. The offer of health actions, their use and the contact through programmed actions were more adequate in the PSF. Key words Primary care, PSF, Assessment of Health policies, Evaluation of the performance of basic health care services
Resumo A pesquisa, desenvolvida dentro dos Estudos de Linha de Base do Proesf analisou o desempenho do Programa Saúde da Família (PSF) em 41 municípios dos Estados de Alagoas, Paraíba, Pernambuco, Piauí, Rio Grande do Norte, Rio Grande do Sul e Santa Catarina. Utilizou delineamento transversal, com grupo de comparação externo (atenção básica tradicional). Entrevistou 41 presidentes de Conselhos Municipais de Saúde, 29 secretários municipais de Saúde e 32 coordenadores de Atenção Básica. Foram caracterizados a estrutura e o processo de trabalho em 234 Unidades Básicas de Saúde (UBS), incluindo 4.749 trabalhadores de saúde; 4.079 crianças; 3.945 mulheres; 4.060 adultos e 4.006 idosos. $O$ controle de qualidade alcançou $6 \%$ dos domicílios amostrados. A cobertura do PSF de 1999 a 2004 cresceu mais no Nordeste do que no Sul. Menos da metade dos trabalhadores ingressaram por concurso público e o trabalho precário foi maior no PSF do que em UBS tradicionais. Os achados sugerem um desempenho da Atenção Básica à Saúde (ABS) ainda distante das prescrições do SUS. Menos da metade da demanda potencial utilizou a UBS de sua área de abrangência. A oferta de ações de saúde, a sua utilização e o contato por ações programáticas foram mais adequados no PSF.

Palavras-chave Atenção Básica, PSF, Avaliação de políticas de saúde, Avaliação de desempenho de serviços básicos de saúde 


\section{Introdução}

A avaliação de políticas e programas é essencial em saúde pública, contribuindo para os esforços em busca de uma sociedade mais saudável e prevenindo o desperdício de recursos com a implementação de programas ineficazes ${ }^{1}$. Na avaliação dos benefícios das políticas de saúde à população, o conhecimento dos arranjos e peculiaridades locais dos serviços de saúde é requisito básico ${ }^{2}$. Entretanto, ao avaliar o efeito de uma política de saúde no desempenho dos serviços e na situação de saúde da população, é preciso valorizar o sinergismo entre os determinantes vinculados à política de saúde, aos serviços de saúde (estruturas, recursos humanos e processos) e ao estado de saúde das populações $3,4,5,6,7$.

Desde o estabelecimento da meta "Saúde para Todos no Ano 2000", em 1978, em AlmaAta $^{8}$, o Brasil implantou o Sistema Único de Saúde (SUS), universalizou o acesso aos serviços e definiu a Atenção Básica à Saúde (ABS) como porta de entrada e principal estratégia para alcançar a meta 9 . Os conceitos de universalidade, integralidade, eqüidade, descentralização e controle social da gestão orientam a ABS para a promoção da saúde, prevenção de agravos, tratamento, reabilitação e manutenção da saúde $8,9,10,11$.

Entretanto, a constituição da ABS no Brasil é marcada por problemas de estrutura física dos serviços, suficiência e perfil dos profissionais de saúde, acesso oportuno aos recursos e efetividade de políticas e ações de saúde 9, 10, 11, 12, 13. Como alternativa, o Programa Saúde da Família (PSF), implantado em 1994, em pequenos municípios do Nordeste, alcança após uma década os grandes centros urbanos de todo o país 10 , 11, 12, 14. O Projeto de Expansão e Consolidação do Saúde da Família (Proesf)15, 16 busca a reformulação da ABS através do PSF. Inicialmente direcionado a municípios com mais de 100 mil habitantes, financia a melhoria da infra-estrutura dos serviços, a capacitação dos recursos humanos e a avaliação do PSF como um requisito obrigatório ${ }^{16}$. Responsável pela avaliação Proesf em municípios das regiões Sul e Nordeste, a Universidade Federal de Pelotas (UFPel) desenvolveu um Estudo de Linha de Base (ELB) com coleta de dados primários e secundários ${ }^{15}$.

Este artigo apresenta os fundamentos do ELB e a avaliação do desempenho da atenção básica à saúde nas duas regiões. O desempenho é avaliado em termos de sua adequação às pres- crições oficiais. Na comparação com o modelo tradicional se examina a plausibilidade de o PSF melhorar o desempenho da atenção básica. $\mathrm{O}$ artigo também identifica alguns pontos prioritários para o desenvolvimento do PSF e da avaliação da atenção básica no País.

\section{Metodologia}

A epidemiologia foi o eixo metodológico do estudo, articulando as dimensões político-institucional, organizacional da atenção, cuidado integral e desempenho do sistema ${ }^{16}$. O delineamento foi transversal, com medidas de múltiplos níveis de agregação ${ }^{17}, 18$. Para a comparação de desempenho dos modelos de atenção, as UBS foram estratificadas em tradicionais e PSF. Foram coletadas informações de fontes primárias e secundárias, que constam da figura 1 .

A dimensão político-institucional reúne aspectos relativos à gestão do SUS, expressando diferenças sociais, econômicas, demográficas e contrastes na constituição da ABS. A dimensão organizacional da atenção examina as práticas de gestão e a oferta de serviços em atenção básica à saúde. A dimensão do cuidado integral avalia os recursos e as práticas dos serviços no cuidado das necessidades de saúde da população. Por fim, a dimensão desempenho do sistema analisa a utilização dos serviços de ABS e a situação de saúde da população.

O universo do estudo foi constituído por 41 municípios com mais de 100 mil habitantes, que compunham os lotes 2 das regiões Sul e Nordeste. Dois municípios eram do Estado de Alagoas, 3 da Paraíba, 10 de Pernambuco, 2 do Piauí, 3 do Rio Grande do Norte, 17 do Rio Grande do Sul e 4 de Santa Catarina16. Uma amostra estratificada por múltiplos estágios selecionou unidades básicas de saúde, profissionais de saúde, usuários e indivíduos residentes na área de abrangência dos serviços 19, 20, 21. Buscando uma maior representatividade, a amostra de UBS foi proporcional ao tamanho da rede básica de saúde de cada município22.

Através dos Projetos de Adesão ao Proesf, foi identificado um total de 855 UBS no Nordeste e 655 UBS no Sul. Em cada lote foram sorteadas aleatoriamente 120 UBS. No Sul, a amostra foi composta por 69 UBS do PSF e 51 Tradicionais, enquanto no Nordeste 79 eram do PSF e 41 Tradicionais. Para facilitar a comparação dos achados, as UBS dos dois modelos foram pareadas, utilizando-se como critério o 


\section{Figura 1}

Modelo teórico para avaliação do desempenho e impacto da Atenção Básica à Saúde em municípios acima de 100 mil habitantes das regiões Sul e Nordeste. Proesf, UFPel, 2005.

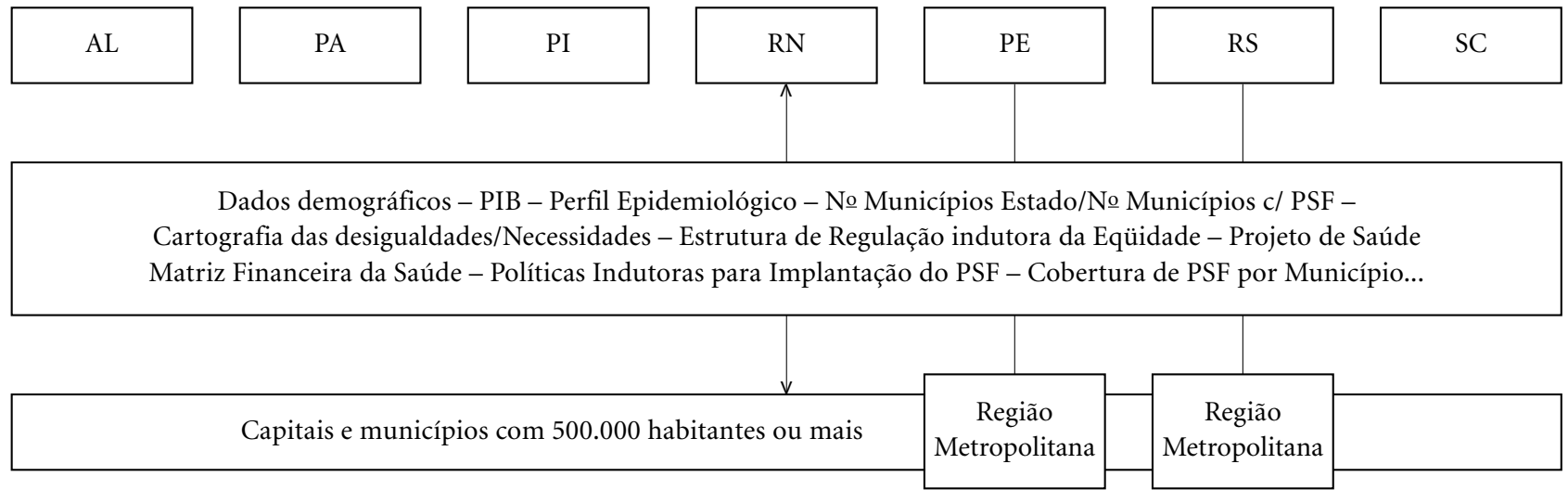

Municípios com população entre 300.000 e 500.000 habitantes

\section{Municípios com população entre 100.000 e 300.000 habitantes}

Unidade Básica de Saúde Programa de Saúde da Família
Unidade Básica de Saúde Tradicional

Estrutura - Processo de Trabalho - Adstrição - Cadastramento - Acolhimento - Vínculo - Ações Programáticas - Integralidade Controle Social - Vigilância Sanitária - Vigilância Epidemiológica - Educação Popular - Educação Permanente

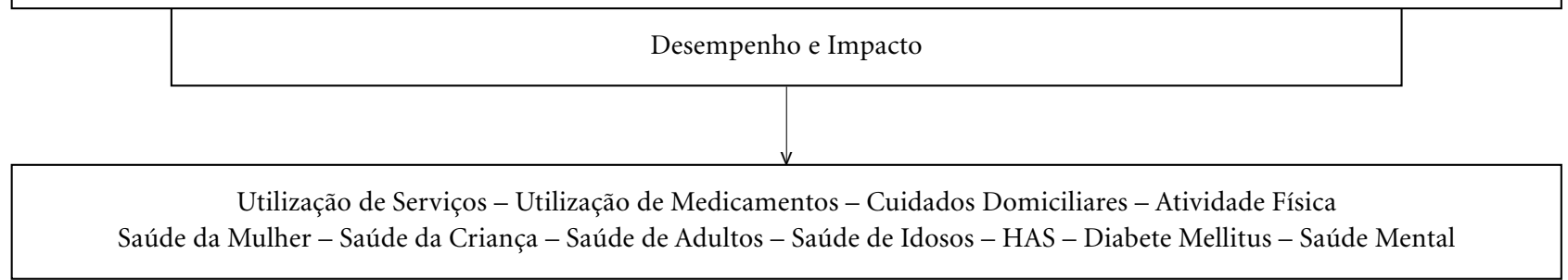

tamanho da área física. As UBS sorteadas orientaram a seleção das amostras de profissionais de saúde, usuários e população.

Em cada UBS procurou-se entrevistar todos os trabalhadores de saúde em atividade. Foram incluídos médicos, enfermeiros, outros profissionais de nível superior, auxiliares de enfermagem, recepcionistas e agentes comunitários de saúde (ACS). A amostra selecionada foi confrontada com o registro de profissionais lotados na UBS obtido a partir do instrumento de avaliação da estrutura das UBS.
A amostra populacional foi dividida em quatro grupos, crianças de um a três anos de idade, mulheres que tiveram filhos nos últimos dois anos, adultos entre 30 e 64 anos de idade e idosos a partir dos 65 anos de idade. Estes indivíduos foram localizados na área de abrangência de cada uma das UBS, ponto inicial para a amostragem sistemática. A estratégia para delimitação da área de abrangência incluiu a obtenção prévia do mapa da área de abrangência das UBS, uma estimativa populacional do IBGE e a localização dos indivíduos através de amostra- 
gem sistemática ${ }^{23}$. Para cada região, estimou-se uma amostra de 2.100 indivíduos em cada um dos grupos populacionais, tamanho suficiente para examinar diferenças de $25 \%$ a $30 \%$ entre os modelos de atenção das UBS (PSF x Tradicional), com um poder estatístico de $80 \%$ e prevalências dos desfechos de no mínimo $25 \%$. Para a composição total da amostra foram estimadas 18 entrevistas de cada grupo populacional na área de abrangência de cada UBS.

O trabalho de campo se estendeu de 15 de março a 19 de maio de 2005 no Sul e de 3 de junho a 10 de agosto de 2005 no Nordeste. As atividades de campo foram realizadas por uma equipe de 15 supervisores criteriosamente selecionados e capacitados para todas as etapas da coleta de dados.

Gestores, coordenadores e profissionais de saúde responderam auto-entrevista, com questões estruturadas e predominantemente fechadas. Os presidentes de CMS foram entrevistados por um supervisor. As informações sobre o processo de trabalho na UBS foram coletadas em reunião da equipe e registradas em instrumento coletivo com questões abertas. Informações sobre a estrutura da UBS foram registradas em instrumento fechado durante reunião de equipe. As informações populacionais, obtidas no domicílio por entrevistadores, foram registradas em questionários estruturados e predominantemente fechados.

Os dados secundários, oriundos de bases nacionais (Ministério da Saúde, DATASUS, IBGE) foram sistematizados, por Estado e município. Os 34 indicadores do Pacto da Atenção Básica formam o primeiro conjunto de informações coletadas 24 . O segundo conjunto de informações inclui os Indicadores de Monitoramento da Expansão do PSF em Grandes Centros Urbanos ${ }^{14}$.

O controle de qualidade realizado por telefone, mediante aplicação de questionários reduzidos, alcançou $6 \%$ dos domicílios no Sul e no Nordeste. No exame da consistência dos dados de variáveis dicotômicas, utilizou-se o índice Kappa e para as quantitativas, o coeficiente de correlação de Spearman ${ }^{25}$. Uma boa repetibilidade (Kappa $>0,7)$ foi observada para a maioria das questões sobre crianças, mulheres, adultos e idosos no Sul e no Nordeste 26 . A repetibilidade satisfatória (acima de 0,7 ) foi maior entre as mulheres $(67 \%)$ em ambas as regiões e menor entre os idosos, principalmente do Nordeste (38\%).

Digitados no EPI-INFO 6.04b, os bancos de dados foram analisados com o pacote estatístico SPSS 10.0 para Windows. Inicialmente, pro- cederam-se às análises descritivas, verificando a distribuição dos casos em cada variável e avaliando sua amplitude e consistência. A seguir, examinou-se o comportamento das variáveis por dimensão teórica e modelo de UBS (Tradicional e PSF). Na análise estratificada do efeito do modelo de UBS, os municípios foram agregados segundo a região (Sul e Nordeste).

Para variáveis dependentes qualitativas, as associações foram testadas através da comparação entre proporções, utilizando-se o teste do qui-quadrado. No caso de variáveis dependentes quantitativas, as associações foram testadas através da comparação entre médias, utilizando-se o teste F (ANOVA). Para ambos os procedimentos, adotou-se o nível de confiança de 95\%, considerando significativas as diferenças com p-valor inferiores a 0,0525. A estratificação possibilitou analisar as diferenças entre os modelos de UBS em ambas as regiões e de seu efeito na distribuição de insumos, no desempenho das UBS e no cuidado à saúde da população ${ }^{4,5,27}$.

\section{Resultados}

\section{Contexto}

Dos 41 municípios estudados, os 21 municípios da região Sul totalizavam 5.423.673 habitantes, enquanto os 20 municípios da região Nordeste somavam 7.575.633 habitantes. No Sul, as médias de Índice de Desenvolvimento Humano (IDH), de expectativa de vida, de alfabetizados e de cobertura de domicílios com água encanada foram mais altas do que no Nordeste, região que apresentava maior proporção de pobres $(41,0 \%)$ e de crianças menores de 5 anos $(9,7 \%)$. No Sul, a maior proporção apresentada foi de idosos (7,7\%). Nas áreas do PSF havia maior proporção de construções inadequadas, de indivíduos com baixa escolaridade e classificados nos estratos econômicos menos privilegiados (D e E) 28 do que nas áreas de UBS Tradicionais. A média de pessoas por domicílio também foi maior nas comunidades do PSF.

\section{Dimensão político-institucional}

\section{- Descentralização da gestão e crescimento do PSF}

A descentralização do SUS, caracterizada através da gestão plena do sistema municipal de saúde, foi significativamente maior no Nor- 
deste (65\% dos municípios) do que no Sul (33\% dos municípios).

O crescimento relativo da cobertura de PSF no período de 1999 a 2004 foi maior no Nordeste do que no Sul. Na amostra estudada, a cobertura de PSF cresceu 35\% ou mais em aproximadamente $65 \%$ dos municípios nordestinos, enquanto, no Sul, apenas 5\% dos municípios experimentaram tal crescimento na cobertura. Em 2004, dez municípios apresentavam cobertura de PSF de no mínimo $60 \%$ da população. Destes, apenas um estava localizado no Sul, enquanto os demais pertenciam à região Nordeste.

O crescimento relativo no número de ESF entre 2003 e 2004 foi duas vezes maior no Sul (44\%) do que no Nordeste (22\%). Mas nos municípios da região Nordeste havia 1.250 ESF, enquanto nos municípios do Sul foram identificadas apenas 358 ESF. Assim, havia uma ESF para 6.060 habitantes no Nordeste; no Sul, a disponibilidade era de uma ESF para 15.149 habitantes.

\section{- Perfil dos profissionais de saúde}

Foram estudados 4.749 profissionais de saúde, 1.730 no Sul (S) e 3.019 no Nordeste (NE). A amostra está composta por 520 médicos $(\mathrm{S}=$ 266; $\mathrm{NE}=254), 337$ enfermeiros $(\mathrm{S}=161 ; \mathrm{NE}=$ 176), 371 profissionais com outros cursos universitários $(S=130 ; \mathrm{NE}=241), 834$ auxiliares e técnicos de enfermagem $(S=414 ; \mathrm{NE}=420)$, 1.045 outros profissionais com nível médio $(\mathrm{S}=261 ; \mathrm{NE}=784)$ e 1.536 agentes comunitários de saúde $(\mathrm{S}=478 ; \mathrm{NE}=1.058)$.

$\mathrm{O}$ ingresso por concurso público alcançou cerca de $40 \%$ dos trabalhadores no Sul e no Nordeste, sendo significativamente maior no modelo Tradicional (50\%) do que no PSF (34\%). O trabalho precário foi significativamente maior no PSF (51\%) do que em UBS Tradicionais $(32 \%)$ na região Nordeste, não havendo diferença entre os modelos no Sul (30\%). A proporção de trabalhadores com outro emprego foi significativamente maior nas unidades Tradicionais (cerca de 30\%) do que no PSF (cerca de $15 \%$ ), independentemente da região.

Nível superior completo foi significativamente maior nas UBS Tradicionais $(\mathrm{S}=44 \%$; $\mathrm{NE}=31 \%$ ) do que no PSF ( $\mathrm{S}=31 \%$; $\mathrm{NE}=26 \%$ ), em ambas as regiões. Em contraste, ensino médio completo foi significativamente maior no PSF ( $\mathrm{S}=38 \%$; $\mathrm{NE}=49 \%$ ) do que no modelo Tradicional $(S=33 \%$; $N E=42 \%)$.
Na composição das ESF do Sul havia 12\% de médicos, $10 \%$ de enfermeiros, $22 \%$ de auxiliares e técnicos de enfermagem, $4 \%$ de dentistas e $36 \%$ de ACS, enquanto nas UBS Tradicionais a proporção de médicos era o dobro, a de enfermeiros e dentistas similar, a de auxiliares e técnicos de enfermagem superior (28\%) e a de ACS 2,6 vezes menor. No Nordeste, as ESF dispunham de $7 \%$ de médicos, $7 \%$ de enfermeiros, $13 \%$ de auxiliares e técnicos de enfermagem, $5 \%$ de dentistas e $44 \%$ de ACS. Nas UBS Tradicionais a proporção de médicos era $11 \%$, a de enfermeiros $4 \%$, a de dentistas similar, a de auxiliares e técnicos de enfermagem superior (16\%) e a de ACS 1,7 vez menor.

\section{Dimensão organizacional da atenção}

\section{- Práticas de gestão}

As práticas de gestão da ABS mais referidas no Sul foram reuniões com a coordenação de distrito ou UBS e com o Conselho Municipal de Saúde, a produção de relatórios de gestão e do SIAB e o acompanhamento dos recursos programados por grupo de procedimentos. No Nordeste foram referidas oficinas de trabalho, cursos de capacitação, atividades de educação permanente, reuniões com o Conselho Municipal de Saúde, as coordenações de outros municípios e de distrito.

A disponibilidade de relatórios periódicos para a tomada de decisão foi pequena no Sul, alcançando pouco mais da metade dos municípios. No Nordeste, 75\% dos municípios referiram dispor de relatórios periódicos, predominantemente mensais ou trimestrais. Os relatórios disponíveis geralmente eram produzidos e divulgados pelas próprias SMS, com limitações de recursos materiais, humanos e escasso apoio ou parceria institucional.

As atividades de supervisão do trabalho nas UBS foram referidas por no máximo $66 \%$ dos trabalhadores de saúde do Sul e 70\% do Nordeste, com predomínio significativo do PSF (77\% no Sul e Nordeste). No Sul, a distância das UBS em relação à sede da Secretaria Municipal de Saúde e as dificuldades operacionais (transporte, pessoal disponível e capacitado, tecnologia da informação) eram grandes desafios para a supervisão periódica e para descentralizar a gestão na atenção básica. No Nordeste, as dificuldades de comunicação com a rede básica foram atribuídas à complexa hierarquia para sua difusão, incluindo diversos níveis de 
gestão (secretário, equipe de assessores, coordenação da atenção básica e PSF, gerência distrital, coordenação de UBS, profissionais de saúde e usuários do SUS).

Do ponto de vista da tecnologia da informação, a situação das UBS era bastante precária. No Sul, $60 \%$ e no Nordeste, $71 \%$ das UBS não dispunham de microcomputador, sem diferença entre os modelos de atenção.

\section{- Práticas de oferta}

Entre as 240 Unidades Básicas de Saúde, obteve-se a avaliação da estrutura para 236 UBS, 118 em cada região. A quase totalidade das UBS (97\%) de ambas as regiões funcionava em dois turnos de atendimento, sem diferença entre os modelos de atenção. No Sul eram atendidas pelo médico do PSF, em média, três pessoas a cada hora, enquanto nas UBS Tradicionais esta média era quatro. No Nordeste eram realizados quatro atendimentos médicos por hora no PSF e cinco nas UBS Tradicionais.

Aproximadamente $96 \%$ das UBS do PSF no Sul e no Nordeste possuíam área geográfica definida e mapa; nas Tradicionais a proporção foi de $51 \%$ no Sul e $33 \%$ no Nordeste. A participação da equipe da UBS em atividades na área de abrangência nos últimos 12 meses foi significativamente maior no PSF ( $63 \%$ no Sul e $71 \%$ no Nordeste) do que nas Tradicionais (36\% no Sul e no Nordeste).

A quase totalidade das UBS (98\%) dispunha de sala de recepção, embora em apenas 13\% dos serviços tenha sido considerada adequada (iluminação, ruído e ventilação). Entre todos os espaços da área física investigados, somente a disponibilidade de sala de reuniões diferiu significativamente com o modelo de UBS. No PSF, cerca de $60 \%$ das UBS do Sul e $69 \%$ do Nordeste dispunham de sala de reuniões, enquanto nas unidades Tradicionais a disponibilidade foi de $36 \%$ no Sul e $44 \%$ no Nordeste.

\section{Dimensão do cuidado integral}

As ações programáticas para o cuidado integral e as atividades de grupo foram significativamente mais freqüentes no PSF do que no modelo Tradicional, tanto no Sul quanto no Nordeste. (Tabela 1)

Os trabalhadores de saúde informaram baixa satisfação com a estrutura física das UBS em ambas as regiões e modelos de atenção. Menos da metade utilizava protocolos em suas ativi- dades, embora significativamente mais no PSF do que no modelo Tradicional tanto no Sul, quanto no Nordeste. Os protocolos citados com maior freqüência foram os de imunização, prénatal, pré-câncer, diagnóstico e tratamento da hipertensão e da tuberculose. (Tabela 1)

\section{Dimensão desempenho da UBS}

Os indicadores de ações preventivas e de promoção da saúde inerentes à Atenção Primária à Saúde e de morbidades específicas na população de abrangência das UBS foram examinados na avaliação do desempenho das duas modalidades de atenção. Estes indicadores também constituem a linha de base para o estudo de acompanhamento, tanto da utilização de serviços básicos de saúde (Tabela 2 ), quanto da situação de saúde da população. (Tabela 3)

\section{- Utilização de serviços: ações de prevenção e de promoção da saúde}

\section{a) Pré-Natal}

A cobertura de pré-natal das 3.945 mulheres que tiveram filho nos últimos dois anos foi de 98\% em ambos os lotes, mas apenas 30\% iniciaram o pré-natal até a 12a semana de gestação, sem diferenças significativas entre as áreas de PSF e Tradicionais. No máximo 50\% das mães realizaram o pré-natal na UBS da área de abrangência de seu domicílio, com proporções significativamente maiores no PSF do que no modelo tradicional nas duas regiões. O número médio de seis ou mais consultas de pré-natal foi alcançado por aproximadamente dois terços das mães que realizaram pré-natal na UBS de sua área, sendo que diferença significativa entre os modelos foi identificada apenas para as usuárias do PSF no Nordeste (74\%) quando comparadas às do modelo Tradicional (64\%). (Tabela 2)

Ter recebido por meio de reuniões ou atividades em grupo o apoio ou suporte para amamentar imediatamente após o parto foi mencionado por aproximadamente um terço das mulheres, exceto nas UBS do PSF do Nordeste, onde alcançou a metade delas. (Tabela 2)

Durante o pré-natal, a vacina contra o tétano deixou de ser administrada em $24 \%$ das mulheres que efetivamente necessitavam, sendo aplicada em excesso em $60 \%$ das gestantes, não diferindo significativamente entre os modelos de UBS, tanto no Sul, quanto no Nordeste. (Tabela 2) 


\section{Tabela 1}

Ações programáticas e processo de trabalho no cuidado integral de saúde segundo o modelo de Atenção Básica em municípios acima de 100 mil habitantes das regiões Sul e Nordeste. Proesf, UFPel, 2005.

\begin{tabular}{|c|c|c|c|c|c|c|}
\hline \multirow[t]{2}{*}{ Indicador } & \multicolumn{3}{|c|}{ Lote Sul } & \multicolumn{3}{|c|}{ Lote Nordeste } \\
\hline & $\begin{array}{l}\text { PSF } \\
\%(\mathrm{n})\end{array}$ & $\begin{array}{c}\text { Tradicional } \\
\%(\mathrm{n})\end{array}$ & $\mathrm{p}$ & $\begin{array}{c}\text { PSF } \\
\%(n)\end{array}$ & $\begin{array}{c}\text { Tradicional } \\
\%(\mathrm{n})\end{array}$ & $\mathrm{p}$ \\
\hline \multicolumn{7}{|l|}{ Atividades Programáticas na UBS } \\
\hline Atendimento odontológico a grupos prioritários & $67,2(67)$ & $57,4(47)$ & & $70,1(77)$ & $63,2(38)$ & \\
\hline Promoção do aleitamento materno & $98,5(68)$ & $65,3(49)$ & * & $97,4(77)$ & $84,6(39)$ & * \\
\hline Puericultura & $98,5(68)$ & $65,3(49)$ & * & $94,7(76)$ & $82,1(39)$ & * \\
\hline Manejo da desnutrição & $80.6(67)$ & $61,2(49)$ & * & $52,0(75)$ & $54,1(37)$ & \\
\hline Manejo dos agravos infantis mais prevalentes & $97,0(66)$ & $71,4(49)$ & * & $84,6(78)$ & $58,3(36)$ & * \\
\hline Pré-natal & $100,0(67)$ & $79,6(49)$ & * & $100,0(77)$ & $97,4(39)$ & \\
\hline Planejamento familiar & $92,6(68)$ & $57,1(49)$ & * & $94,8(77)$ & $84,6(39)$ & \\
\hline Prevenção do CA de colo uterino & $98,5(68)$ & $84,0(50)$ & * & $93,6(78)$ & $92,3(39)$ & \\
\hline \multicolumn{7}{|l|}{ Diagnóstico e tratamento } \\
\hline Tuberculose & $53,0(66)$ & $21,3(47)$ & $\star$ & $91,0(78)$ & $48,7(39)$ & * \\
\hline Hanseníase & $39,4(66)$ & $10,4(48)$ & * & $87,0(77)$ & $33,3(39)$ & * \\
\hline Hipertensão arterial sistêmica & $97,1(68)$ & $95,4(49)$ & & $100,0(77)$ & $94,6(39)$ & * \\
\hline Diabetes & $100,0(67)$ & $91,8(49)$ & * & $100,0(78)$ & $92,3(39)$ & * \\
\hline Cuidado/visita domiciliar & $98,7(68)$ & $95,6(48)$ & * & $41,7(77)$ & $43,6(39)$ & * \\
\hline Atividades de grupo & $91,0(68)$ & $63,8(47)$ & * & $91,0(78)$ & $64,9(37)$ & * \\
\hline \multicolumn{7}{|l|}{ Satisfação profissional de 0 a 10} \\
\hline Estrutura da UBS & $5,4(1048)$ & $5,4(640)$ & & $5,6(1592)$ & $4,7(1341)$ & * \\
\hline \multicolumn{7}{|l|}{ Processo de Trabalho na UBS } \\
\hline \multicolumn{7}{|l|}{ Protocolos que orientem as atividades } \\
\hline$\%$ de profissionais que utilizam & $39,2(1018)$ & $31,6(604)$ & * & $50,5(1544)$ & $44,6(1290)$ & * \\
\hline \multicolumn{7}{|l|}{ Protocolos mais utilizados } \\
\hline 10 & $\begin{array}{c}\text { Imunização } \\
(85,1)\end{array}$ & $\begin{array}{c}\text { Pré-câncer } \\
(79,6)\end{array}$ & & $\begin{array}{c}\text { Imunização } \\
(93,5)\end{array}$ & $\begin{array}{c}\text { Imunização } \\
(91,9)\end{array}$ & \\
\hline 20 & $\begin{array}{c}\text { Pré-natal } \\
(79,1)\end{array}$ & $\begin{array}{c}\text { Imunização } \\
(74,5)\end{array}$ & & $\begin{array}{c}\text { Pré-natal } \\
(86,8)\end{array}$ & $\begin{array}{c}\text { Pré-natal } \\
(84,2)\end{array}$ & \\
\hline 30 & $\begin{array}{c}\text { Pré-câncer } \\
(71,6)\end{array}$ & $\begin{array}{l}\text { HAS } \\
(71,4)\end{array}$ & & $\begin{array}{c}\text { Tuberculose } \\
(85,7)\end{array}$ & $\begin{array}{l}\text { Pré-câncer } \\
\quad(81,6)\end{array}$ & \\
\hline
\end{tabular}

${ }^{*} \mathrm{p}$ valor $<0,05$

Cerca de $80 \%$ das entrevistadas que realizaram o pré-natal na UBS da área de abrangência expressaram opinião positiva sobre o programa. Essa opinião foi significativamente melhor no PSF do que no modelo tradicional nas duas regiões. (Tabela 2)

\section{b) Puericultura}

A realização de puericultura foi referida para $91 \%$ das 4.079 crianças estudadas, a metade das quais atendida na UBS da área de abrangência, sendo significativamente superior apenas no PSF do Nordeste. Entre as crianças usuárias da UBS da área de abrangência, dois terços fizeram, no mínimo, sete acompanhamentos de puericultura, também sendo significativa- mente superior no PSF do Nordeste. A cobertura vacinal completa para Sabin, Sarampo, BCG, Hepatite e Tetravalente, coletada a partir do cartão de vacinação e, em sua ausência, da informação materna, foi superior a $80 \%$, sem diferenças significativas entre regiões e modelos de UBS. Registrou-se, no máximo, 10\% de informação ignorada para as vacinas avaliadas. A opinião sobre a puericultura foi boa, muito boa ou ótima para dois terços das mães entrevistadas, sendo significativamente maior $(82 \%)$ nas UBS do PSF do Nordeste. (Tabela 2) 
Tabela 2

Utilização de serviços de saúde: ações preventivas e de promoção da saúde segundo o Modelo de Atenção Básica em 41 municípios acima de 100 mil habitantes das regiões Sul e Nordeste. Proesf, UFPel, 2005.

\begin{tabular}{|c|c|c|c|c|c|c|}
\hline $\begin{array}{l}\text { Indicador } \\
\text { Ações preventivas e de promoção da saúde (\%) }\end{array}$ & $\begin{array}{l}\text { PSF } \\
\%(n)\end{array}$ & $\begin{array}{c}\text { Lote Sul } \\
\text { Tradicional } \\
\%(\mathrm{n})\end{array}$ & $\mathrm{p}$ & $\begin{array}{l}\text { PSF } \\
\%(n)\end{array}$ & $\begin{array}{c}\text { Lote Nordeste } \\
\text { Tradicional } \\
\%(n)\end{array}$ & $\mathrm{p}$ \\
\hline \multicolumn{7}{|l|}{ Mulheres com filho nos últimos 2 anos } \\
\hline Pré-natal na última gestação & $97,0(1051)$ & $97,8(774)$ & & $97,1(1360)$ & $97,9(752)$ & \\
\hline Iniciou pré-natal até 12 semanas de gestação & $23,6(890)$ & $26,0(685)$ & & $32,5(1109)$ & $34,9(627)$ & \\
\hline Pré-natal na UBS da área & $47,9(1019)$ & $39,7(748)$ & * & $55,5(1315)$ & $46,3(722)$ & * \\
\hline Fez 6 ou + consultas de pré-natal na UBS da área & $79,3(484)$ & $74,0(296)$ & & $74,0(730)$ & $64,3(334)$ & * \\
\hline Recebeu apoio ao aleitamento materno no pós-parto & $28,6(486)$ & $19,1(293)$ & * & $50,0(726)$ & $36,4(332)$ & * \\
\hline Com vacinação antitetânica em excesso & $57,5(557)$ & $58,8(444)$ & & $63,8(840)$ & $59,5(439)$ & \\
\hline Com vacinação antitetânica incompleta & $28,7(244)$ & $32,8(201)$ & & $16,2(308)$ & $20,9(163)$ & \\
\hline Pré-natal "bom / muito bom" na UBS da área & $83,3(485)$ & $76,1(289)$ & * & $85,2(728)$ & $80,3(330)$ & * \\
\hline Conheciam o exame citopatológico & $89,6(1051)$ & $90,8(774)$ & & $90,1(1359)$ & $95,1(753)$ & * \\
\hline Fez pelo menos 1 exame citopatológico na vida & $82,1(941)$ & $82,8(702)$ & & $83,3(1224)$ & $82,5(716)$ & \\
\hline \multicolumn{7}{|l|}{ Crianças de 1 a 3 anos de idade } \\
\hline Com puericultura & $91,3(1132)$ & $90,9(817)$ & & $91,8(1411)$ & $90,1(718)$ & \\
\hline Puericultura na UBS da Área & $54,3(1112)$ & $50,2(779)$ & & $54,5(1377)$ & $44,7(702)$ & * \\
\hline Com 7 ou + consultas de puericultura na UBS da área & $69,7(1034)$ & $68,8(737)$ & & $76,0(1296)$ & $70,0(647)$ & * \\
\hline Crianças com cobertura vacinal completa & $71,8(964)$ & $76,7(647)$ & * & $79,8(1310)$ & $81,0(654)$ & \\
\hline Puericultura "boa / muito boa" na UBS da área & $76,2(608)$ & $75,2(403)$ & & $82,3(776)$ & $74,7(320)$ & * \\
\hline \multicolumn{7}{|l|}{ Adultos de 30 a 64 anos } \\
\hline $\begin{array}{l}\text { Recomendação médica de atividade física na UBS } \\
\text { alguma vez na vida }\end{array}$ & $29,4(883)$ & $22,6(646)$ & * & $34,4(1125)$ & $24,3(584)$ & * \\
\hline $\begin{array}{l}\text { Recomendação médica de atividade física na UBS } \\
\text { na última consulta }\end{array}$ & $23,8(883)$ & $16,8(668)$ & * & $29,7(1161)$ & $20,3(566)$ & * \\
\hline Mulheres com pelo menos 1 exame citopatológico na vida & $92,6(619)$ & $94,3(440)$ & & $93,1(726)$ & $92,0(351)$ & \\
\hline \multicolumn{7}{|l|}{ Idosos de 65 ou + anos } \\
\hline $\begin{array}{l}\text { Recomendação médica de atividade física na UBS } \\
\text { alguma vez na vida }\end{array}$ & $38,7(938)$ & $31,3(667)$ & * & $45,2(1189)$ & $35,1(601)$ & * \\
\hline $\begin{array}{l}\text { Recomendação médica de atividade física na UBS } \\
\text { na última consulta }\end{array}$ & $30,7(919)$ & $22,9(650)$ & * & $35,6(1178)$ & $27,2(591)$ & * \\
\hline Idosos com necessidade de cuidado domiciliar regular & $18,2(1085)$ & $10,5(784)$ & * & $25,2(1404)$ & $23,5(702)$ & \\
\hline $\begin{array}{l}\text { Idosos que receberam cuidado domiciliar nos últimos } \\
3 \text { meses }\end{array}$ & $13,3(1096)$ & $2,9(790)$ & * & $20,1(1407)$ & $8,0(703)$ & * \\
\hline
\end{tabular}

${ }^{*} \mathrm{p}$ valor $<0,05$

\section{c) Recomendação de atividade física para adultos e idosos}

A recomendação de exercício físico para melhorar a saúde feita por médico da UBS da área foi referida por aproximadamente um terço dos adultos, e por pouco menos da metade dos idosos, sendo significativamente maior no PSF. (Tabela 2)

\section{d) Realização de exame citopatológico por mulheres de 30 a 64 anos}

Entre as 2.238 mulheres de 30 a 64 anos de idade, $93 \%$ referiram ter realizado pelo menos um exame citopatológico na vida, sem diferença entre os modelos de UBS. (Tabela 2)

\section{e) Cuidado domiciliar de idosos}

Na região Sul a necessidade de cuidados domiciliares com regularidade foi referida por um quinto dos idosos das unidades do PSF e 


\section{Tabela 3}

Situação de saúde na área de abrangência da UBS segundo o Modelo de Atenção Básica em municípios acima de 100 mil habitantes das regiões Sul e Nordeste. Proesf, UFPel, 2005.

\begin{tabular}{|c|c|c|c|c|c|c|}
\hline \multirow[t]{2}{*}{ Indicador } & \multicolumn{3}{|c|}{ Lote Sul } & \multicolumn{3}{|c|}{ Lote Nordeste } \\
\hline & $\begin{array}{c}\text { PSF } \\
\%(\mathrm{~N})\end{array}$ & $\begin{array}{l}\text { Tradicional } \\
\%(\mathrm{~N})\end{array}$ & $\mathrm{p}$ & $\begin{array}{c}\text { PSF } \\
\%(\mathrm{~N})\end{array}$ & $\begin{array}{l}\text { Tradicional } \\
\%(\mathrm{~N})\end{array}$ & $\mathrm{p}$ \\
\hline \multicolumn{7}{|l|}{ Morbidades (\%) } \\
\hline \multicolumn{7}{|l|}{ Diarréia em crianças } \\
\hline Com diarréia no último mês & $25,0(1127)$ & $19,5(815)$ & * & $36,3(1403)$ & $38,6(717)$ & \\
\hline Consultaram por diarréia & $53,2(284)$ & $51,3(160)$ & & $51,9(513)$ & $52,9(276)$ & \\
\hline Consultaram por diarréia na UBS da área & $48,1(154)$ & $45,1(82)$ & & $53,0(270)$ & $29,7(148)$ & * \\
\hline \multicolumn{7}{|l|}{ Pneumonia em crianças } \\
\hline Com pneumonia nos últimos 6 meses & $6,6(1131)$ & $6,09(814)$ & & $7,4(1410)$ & $7,9(718)$ & \\
\hline Consultaram por pneumonia & $97,3(73)$ & $100,0(49)$ & & $97,6(101)$ & $92,9(56)$ & \\
\hline Consultaram por pneumonia na UBS da área & $36,6(71)$ & $26,5(49)$ & & $27,3(99)$ & $15,4(52)$ & \\
\hline \multicolumn{7}{|l|}{$\begin{array}{l}\text { Situação de Saúde: autopercepção excelente / } \\
\text { muito boa /boa }\end{array}$} \\
\hline Adultos & $56,3(1124)$ & $61,7(808)$ & & $43,7(1426)$ & $46,5(690)$ & \\
\hline Idosos & $38,1(1089)$ & $40,8(791)$ & & $32,9(1381)$ & $30,7(690)$ & \\
\hline \multicolumn{7}{|l|}{ Hipertensão arterial sistêmica (HAS) referida } \\
\hline \multicolumn{7}{|l|}{ Adultos } \\
\hline Com HAS & $29,3(1100)$ & $26,2(801)$ & & $28,8(1384)$ & $26,7(675)$ & \\
\hline Consultaram na UBS da área nos últimos 6 meses & $46,5(314)$ & $31,4(207)$ & * & $56,1(383)$ & $38,4(177)$ & * \\
\hline \multicolumn{7}{|l|}{ Idosos } \\
\hline Com HAS & $64,3(1091)$ & $58,4(791)$ & * & $65,3(1388)$ & $64,2(693)$ & \\
\hline Consultaram na UBS da área nos últimos 6 meses & $49,8(663)$ & $37,1(447)$ & * & $52,6(863)$ & $34,9(435)$ & * \\
\hline \multicolumn{7}{|l|}{ Diabetes mellitus (DM) referido } \\
\hline \multicolumn{7}{|l|}{ Adultos } \\
\hline Com DM & $6,2(1092)$ & $7,6(802)$ & & $7,0(1358)$ & $6,0(645)$ & \\
\hline Consultaram na UBS da área nos últimos 6 meses & $57,6(66)$ & $39,3(61)$ & * & $56,4(94)$ & $39,5(38)$ & \\
\hline \multicolumn{7}{|l|}{ Idosos } \\
\hline Com DM & $22,0(1083)$ & $17,8(780)$ & * & $19,0(2046)$ & $18,4(681)$ & \\
\hline Consultaram na UBS da área nos últimos 6 meses & $52,7(224)$ & $38,8(134)$ & * & $48,0(246)$ & $35,9(117)$ & * \\
\hline \multicolumn{7}{|l|}{ Problema de nervos referido } \\
\hline \multicolumn{7}{|l|}{ Adultos } \\
\hline Com problemas de nervos & $29,6(1120)$ & $25,5(811)$ & & $23,8(1414)$ & $25,3(688)$ & \\
\hline Consultaram na UBS da área nos últimos 6 meses & $21,5(326)$ & $15,1(205)$ & & $16,1(335)$ & $7,6(171)$ & * \\
\hline \multicolumn{7}{|l|}{ Idosos } \\
\hline Com problema de nervos & $35,2(1092)$ & $31,4(794)$ & & $27,5(1389)$ & $19,5(681)$ & * \\
\hline Consultaram na UBS da área nos últimos 6 meses & $16,8(376)$ & $15,2(244)$ & * & $14,6(369)$ & $11,7(132)$ & \\
\hline
\end{tabular}

$10 \%$ das unidades Tradicionais $(\mathrm{p}<0,05)$. Nas áreas do PSF, $13 \%$ receberam este cuidado e nas áreas Tradicionais apenas 3\% $(\mathrm{p}<0,05)$. No Nordeste a necessidade foi informada por um quarto dos idosos de ambos os modelos de UBS e o cuidado foi recebido por $20 \%$ dos idosos nas áreas do PSF e $8 \%$ nas áreas Tradicionais $(\mathrm{p}<0,05)$. (Tabela 2$)$

\section{- Situação de saúde na área de abrangência das UBS}

\section{a) Diarréia em crianças}

A ocorrência de diarréia no mês anterior à entrevista foi informada para $30 \%$ das crianças, sendo no Sul significativamente maior no PSF (25\%) do que nas Tradicionais (20\%). Destas crianças, pouco mais da metade se consultou $(52 \%)$ em ambas as regiões, sem diferença 
entre os modelos de UBS. Menos da metade das consultas (46\%) foi na UBS da área de abrangência, sendo maior a proporção no PSF do Nordeste (53\%). (Tabela 3)

\section{b) Pneumonia em crianças}

Para o período dos seis meses anteriores à entrevista, a ocorrência de pneumonia foi referida para 7\% das crianças, sem diferença significativa por modelo de UBS. A quase totalidade das crianças (97\%) consultou pela pneumonia, com distribuição semelhante entre os modelos de UBS. Apenas $27 \%$ das crianças foram atendidas na UBS de sua área geográfica, sem diferença segundo o modelo de UBS. (Tabela 3)

\section{c) Situação de saúde de adultos e idosos}

Aproximadamente 50\% dos 4.060 adultos e $36 \%$ dos 4.006 idosos identificaram sua situação de saúde como excelente, muito boa ou boa, sem diferença entre os modelos de atenção. (Tabela 3)

\section{d) Hipertensão arterial sistêmica em adultos e idosos}

O diagnóstico de HAS foi informado por $28 \%$ dos adultos e $63 \%$ dos idosos entrevistados, sem diferença significativa entre os modelos de atenção, com exceção de idosos de áreas do PSF no Sul (64\%). O tempo médio de conhecimento da HAS foi de 7,4 anos $(\mathrm{dp}=7,8)$ para os adultos e 10,9 anos $(\mathrm{dp}=10,5)$ para os idosos, sem variação significativa entre os modelos de UBS. Consulta por HAS na UBS da área nos últimos seis meses foi informada por $46 \%$ dos adultos e dos idosos, sendo significativamente maior no PSF do que nas UBS Tradicionais. (Tabela 3)

\section{e) Diabetes mellitus em adultos e idosos}

O diagnóstico de diabetes mellitus (DM) foi informado por $7 \%$ dos adultos e $20 \%$ dos idosos, não havendo diferenças significativas entre os modelos de atenção, com exceção de idosos de áreas do PSF no Sul (22\%). O tempo médio de conhecimento do diagnóstico foi de 5,9 anos $(\mathrm{dp}=6,2)$ para adultos e 9,2 anos $(\mathrm{dp}=$ $9,4)$ para idosos, sem diferença entre os modelos de UBS. Cerca de $50 \%$ dos adultos e $46 \%$ dos idosos com diabete consultaram, por este problema, na UBS da área. Para os adultos, essa proporção foi significativamente maior nas unidades do PSF do Sul (58\%); para os idosos, o acesso foi maior no PSF tanto do Sul (53\%), quanto do Nordeste (48\%) (Tabela 3).

\section{f) Problemas de nervos em adultos e idosos}

O padecimento de "problemas de nervos" foi referido por $26 \%$ dos adultos e $29 \%$ dos idosos. Entre os adultos, não houve diferença entre os modelos, mas entre os idosos, a ocorrência foi significativamente menor em áreas de UBS Tradicional do Nordeste (20\%). A média de duração deste sofrimento foi de 12,6 anos $(\mathrm{dp}=11,5)$ para os adultos e 19,1 anos $(\mathrm{dp}=17,8)$ para os idosos, não variando por modelo de UBS. Apenas 16\% dos adultos e 15\% dos idosos com "problemas de nervos" consultaram na UBS da área por estes problemas nos últimos seis meses. No Nordeste, o acesso a este cuidado foi significativamente maior para adultos no PSF (16\%) do que nas UBS Tradicionais $(8 \%)$. (Tabela 3 )

\section{Discussão}

\section{Avaliação metodológica}

O delineamento do estudo foi orientado para o exame de uma intervenção em saúde pública complexa, programática e dependente do contexto 29 . O estudo foi planejado para avaliar o desempenho da ABS no lote de municípios, não sendo seus achados generalizáveis para o contexto municipal 17 .

Estudos observacionais criteriosos podem ser vantajosos em relação aos ensaios clínicos na avaliação de intervenções em saúde pública18, 27, 30,31. O estabelecimento de grupos de comparação, a composição de uma amostra de múltiplos níveis de análise, a realização de medidas padronizadas e a utilização de critérios bem definidos para julgar os achados reforçam a adequação do ELB. Estudos com estas características têm maior capacidade de detectar o sucesso ou a falha da intervenção, aumentando a validade dos resultados, sendo, portanto, recomendáveis na avaliação de políticas e programas de saúde17, 27, 29, 30,32.

A coleta de dados primários em diversos níveis de avaliação (gestão, unidades de saúde e população), através de instrumentos padronizados, permite análises mais sofisticadas do desempenho dos serviços de atenção básica à saú- 
de 31 . De um modo geral, as amostras populacionais foram suficientes para examinar diferenças socioeconômicas, demográficas e de utilização de serviços, permitindo comparações regionais e por modelo de UBS33.

O controle de qualidade realizado por telefone desde a sede do estudo mostrou, de um modo geral, uma repetibilidade aceitável, similar a de estudos de grande complexidade 34,35 .

Além disso, a baixa repetibilidade em idosos pode estar mais relacionada à estratégia de controle de qualidade ${ }^{35}$, do que à validade do dado primário, obtido no domicílio do entrevistado, por auxiliar de pesquisa capacitado. Mediante telefonemas realizados da sede do estudo, uma entrevistadora interagia com os idosos de outras regiões e Estados do País, enfrentando desde problemas auditivos, viés de memória e dificuldades de comunicação, até contrastes culturais e de diferenças idiomáticas. Diferenças na cobertura de telefone, associadas à dificuldade de locomoção de parte dos idosos também prejudicaram o controle de qualidade. Assim, o estudo ao avaliar a qualidade dos dados primários por telefone, acabou testando uma estratégia de controle de qualidade que pode ser desenvolvida em nosso meio 35,36 .

O aprimoramento das características metodológicas do ELB deverá potencializar sua utilização tanto na avaliação da ABS como uma intervenção política em saúde pública, quanto na avaliação de uma ação programática em uma UBS particular ${ }^{31}$

\section{Avaliação da implantação do PSF}

A implantação do PSF estava mais consolidada nos grandes centros urbanos do Nordeste do que no Sul do país 14, 16. O PSF ainda é uma estratégia em construção, carecendo de melhor definição em aspectos conceituais e operacionais 12,14 . No ano de 2000, a totalidade dos municípios estudados na região Nordeste já dispunha de ESF, enquanto no Sul, principalmente no interior do Rio Grande do Sul, a expansão do PSF foi recente, estando mais fortemente associada ao Proesf.

Embora as responsabilidades municipais na gestão da $\mathrm{ABS}$ sejam requisito da descentralização do SUS10, 11, sua efetivação ainda é muito problemática ${ }^{37}$. Na maioria dos municípios, o monitoramento e a avaliação da atenção básica à saúde era irregular e materialmente desestruturado. A supervisão era de caráter informativo, com vistas a repassar aos profissionais de saúde e coordenadores de UBS normas e procedimentos burocráticos, geralmente associados ao faturamento do SUS ou a reclamações de usuários e da mídia. A utilização de relatórios periódicos para a tomada de decisão foi rara, devido à sobrecarga de trabalho das equipes nos municípios e à necessidade de capacitação para as atividades de monitoramento e avaliação. O trabalho burocrático nas UBS raramente estava informatizado, ocupando parte importante do tempo dos profissionais de saúde, em detrimento de suas atividade-fins ${ }^{38}, 39$. Portanto, a gestão da ABS necessita de um forte estímulo para seu desenvolvimento, não apenas em termos materiais, mas particularmente de capacitação de gestores e de estratégias gerenciais focadas nos objetivos prioritários.

A delimitação de área e população de abrangência para a oferta de serviços no PSF11 representa um avanço programático, em relação ao padrão de "população aberta" adotado no modelo tradicional. Esta estratégia facilita a disponibilidade regular de unidades básicas de saúde à população, condição para o acesso aos cuidados de saúde 33 . O PSF amplia a equipe de saúde, com a inserção obrigatória do enfermeiro e do ACS11, o que pode contribuir para o desempenho da UBS. Por outro lado, a maior produtividade médica no atendimento individual das UBS tradicionais não garantiu um melhor desempenho ao modelo. Enquanto as UBS tradicionais estavam mais focadas no atendimento à demanda, o PSF estava mais voltado a ações programáticas, atividades domiciliares e, articulação com a comunidade11,12, 40. Portanto, a oferta de ações e recursos de saúde foi mais adequada no PSF do que no modelo tradicional.

\section{Avaliação da adequação e da plausibilidade do desempenho do PSF}

O desempenho do PSF foi regularmente melhor do que o de serviços tradicionais, tanto no Sul, quanto no Nordeste. Este achado coincide com a literatura12, 13, 40 e com a percepção dos gestores de que o PSF é mais adequado para o funcionamento do sistema municipal de saúde e conta com uma maior adesão dos profissionais de saúde do que o modelo tradicional15.

O PSF representou um esforço bem-sucedido de promoção da eqüidade, pois normalmente sua presença era maior em regiões mais pobres e com população mais vulnerável. Seu melhor desempenho em contextos de maior iniqüidade social e de saúde reforça o efeito do 
PSF na melhoria da atenção básica à saúde no país. Apesar de suas limitações, comuns aos modelos de ABS, o PSF faz mais para quem mais precisa 33 .

Na região Sul, o PSF necessita de uma forte expansão para alcançar uma cobertura populacional de $60 \%$, conforme estabelecido pelo Proesf 16 , enquanto no Nordeste é preciso consolidar o aumento crescente da cobertura. Neste processo, a maior precarização do trabalho promovida pelo PSF, principalmente no Nordeste, representa um aspecto político da estratégia que deve ser enfrentado, para não comprometer seus benefícios.

A estrutura física das UBS foi bastante similar nas duas modalidades de atenção básica, destacando-se por sua precariedade e improvisação40. A maior disponibilidade de sala de reunião no PSF significa mais espaço na UBS para atividades que não estão centradas no médico e no usuário individual, podendo contribuir para o melhor desempenho do modelo em relação às ações programáticas e gestão dos serviços 3,41 .

A equipe do PSF tem um perfil ocupacional bastante distinto da equipe tradicional11, sendo profissionalmente mais diversificada e podendo contribuir para o melhor desempenho do novo modelo 3,40 .

\section{Colaboradores}

LA Facchini, RX Piccini, E Tomasi, E Thumé e D Silveira participaram da concepção, análise de dados, interpretação e redação final do artigo. FV Siqueira coordenou o trabalho de campo do estudo e participou da elaboração dos instrumentos e processamento de dados. MA Rodrigues participou do processamento de dados, elaboração dos instrumentos e revisão da literatura.

\section{Agradecimentos}

Os autores agradecem aos gestores, coordenadores de atenção básica e de saúde da família, representantes do controle social, trabalhadores das unidades básicas de saúde e à população entrevistada o apoio para a realização deste estudo. Agradecem também aos supervisores do trabalho de campo e a todos aqueles que trabalharam nas diversas etapas do estudo. O presente artigo integra o Componente 3 do Projeto de Expansão e Consolidação do Saúde da Família (Proesf) financiado pelo Ministério da Saúde e Banco Mundial.
Em conseqüência, a utilização de serviços básicos de saúde foi mais adequada no PSF do que em UBS Tradicionais para o conjunto dos grupos populacionais, confirmando indícios observados em estudos anteriores $12,13,40$.

Para maior benefício da população e melhor desempenho do PSF diante das metas de Alma-Ata, há necessidade de estímulo financeiro, técnico e político à rede básica de saúde no país. Os investimentos em infra-estrutura são urgentes e precisam financiar a reforma e a construção de UBS na concepção do PSF e dos requisitos legais de conforto e segurança ambientais. A Educação Permanente de Profissionais de Saúde requer investimentos orientados para o desempenho dos serviços e a vulnerabilidade social dos problemas de saúde. Assim, será mais fácil direcionar as práticas na UBS para o monitoramento e acompanhamento criterioso de indivíduos mais vulneráveis. A implantação de grupos locais em avaliação e monitoramento de saúde, que inclua a capacitação de profissionais das UBS, será benéfica a Estados e municípios, requerendo recursos materiais e humanos para sua efetivação ${ }^{33}$. O adequado enfrentamento dos principais problemas do PSF poderá incrementar significativamente seu desempenho e impacto positivo na saúde da população.

\section{Referências}

1. Vaughan R. Evaluation and public health. Am J Publ Health 2004; 94(3):360.

2. Hartz ZMA, Contandriopoulos AP. Integralidade da atenção e integração de serviços de saúde: desafios para avaliar a implantação de um sistema sem muros. Cad Saúde Pública 2004; 20(Supl. 2):331-36.

3. Donabedian A. The effectiveness of quality assurance. Int J Qual Health Care 1996; 8(4):401-7.

4. Shaw M, Tunstall H, Smith GD. Seeing social position: visualizing class in life and death. Int J Epidemiol 2003; 32(3):332-5.

5. Stafford M, Marmot M. Neighbourhood deprivation and health: does it affect us all equally? Int J Epidemiol 2003; 32(3):357-66.

6. Viacava F, Almeida C, Caetano R, Fausto M, Masinko J, Martins $M$ et al. Uma metodologia de avaliação do desempenho do sistema de saúde brasileiro. Rev C S Col 2004; 9(3):711-24.

7. Victora CG, Habicht JP, Bryce J. Evidence-based public health: moving beyond randomized trials. Am J Publ Health 2004; 94(3):400-5.

8. Organización Mundial de la Salud. Saúde para todos no ano 2000. Geneva: OMS; 1978. 
9. Brasil. Lei no 8.080, de 19 de setembro de 1990: dispõe sobre as condições para a promoção, proteção e recuperação da saúde, a organização e o funcionamento dos serviços correspondentes, e dá outras providências. Brasília: Congresso Nacional; 1990.

10. Brasil. Ministério da Saúde. Regionalização da Assistência à Saúde: Aprofundando a Descentralização com Eqüidade no Acesso (Norma Operacional da Assistência à Saúde. NOAS. SUS 01/01. Portaria MS/ GM no 95, de 26 de Janeiro de 2001). Brasília: MS; 2001.

11. Brasil. Ministério da Saúde. Guia Prático do Programa Saúde da Família. Brasília: MS; 2001.

12. Conill EM. Políticas de atenção primária e reformas sanitárias: discutindo a avaliação a partir da análise do Programa Saúde da Família em Florianópolis, Santa Catarina, Brasil, 1994-2000. Cad Saúde Pública 2002; 18(Suppl):191-202.

13. Tomasi E, Facchini LA, Osorio A, Fassa AG. Aplicativo para sistematizar informações no planejamento de ações de saúde pública. Rev Saúde Publ 2003; 37(6):800-6.

14. Viana ALdÁ, Heimann LS, Lima LD, Oliveira RG, Rodrigues SH. Mudanças significativas no processo de descentralização do sistema de saúde no Brasil. Cad Saúde Pública 2002; 18(Supl):139-51.

15. Facchini LA, Piccini RX, Tomasi E, Thumé E, Silveira DS. Projeto de Monitoramento e Avaliação do Programa de Expansão e Consolidação do Saúde da Família (Proesf). Pelotas: UFPel; 2006.

16. Brasil. Ministério da Saúde. Termo de referência para o estudo de linha de base nos municípios selecionados para o componente 1 do Proesf. Brasília: MS; 2004.

17. Rothman K, Greenland S. Modern epidemiology. Philadelphia: Lippincott-Raven; 1998.

18. Santos IS, Victora CG. Serviços de saúde: epidemiologia, pesquisa e avaliação. Cad Saúde Pública 2004; 20(Supl. 2):337-41.

19. Lemeshow S, Hosmer D. Adequacy of sample size in health studies. Chichester: John Wiley; 1990.

20. Levy P, Lemeshow S. Sampling for health professionals. Belmont: LLP; 1980.

21. Lwanga S, Lemeshow S. Sample size determination in health studies: a practical manual. Geneva: WHO; 1991.

22. Barros FC, Victora CG. Epidemiologia da saúde infantil. 3 ed. São Paulo: Hucitec; 1998.

23. Instituto Brasileiro de Geografia e Estatística [acesso em jan. 2006]. Acesso e utilização de serviços de saúde: IBGE; 2003. Disponível em: http://www.ibge.gov. br/home/estatistica/populacao/trabalhoerendimento/pnad2003/saude/default.shtm>.

24. Brasil. Ministério da Saúde. Secretaria de Atenção à Saúde, Departamento de Atenção Básica. Pacto de indicadores da atenção básica: instrumento de negociação qualificador do processo de gestão do SUS. Rev Bras Saúde Mat. Infant. 2003; 3(2):221-224.

25. Kirkwwod BR. Essentials of medical statistics. Oxford: Blackwell; 1988.
26. Landis JR, Koch GG. The measurement of observer agreement for categorical data. Biometrics 1977; 33(1):159-74.

27. Des Jarlais DC, Lyles C, Crepaz N. Improving the reporting quality of nonrandomized evaluations of behavioral and public health interventions: the TREND statement. Am J Publ Health 2004; 94(3):361-6.

28. Associação Brasileira de Empresas de Pesquisa. Critério de classificação econômica Brasil. São Paulo: ABEP; 2003. [acessado em fev. 2006]. Disponível em: http://www.abep.org/codigosguias/ABEP_CCEB.pdf.

29. Rychetnik L, Frommer M, Hawe P, Shiell A. Criteria for evaluating evidence on public health interventions. J. Epidemiol. Community Health 2002; 56:119-27.

30. Black D, Morris J, Smith C, Townsend P. Inequalities in health: report of a Research Working Group. Washington: National Academy Press; 1993.

31. Heller RF, Page J. A population perspective to evidence based medicine: "evidence for population health". J Epidemiol Community Health 2002; 56(1): 45-7.

32. Habicht JP, Victora CG, Vaughan JP. Evaluation designs for adequacy, plausibility and probability of public health programme performance and impact. Int J Epidemiol 1999; 28(1):10-8.

33. Millman M, editor. Access to health care in America. Washington, DC: National Academy Press; 1993.

34. Aquilino WS. Telephone versus face-to-face interviewing for household drug use serveys. Int J Addict 1992; 27(1):71-91.

35. Voigt LF, Koepsell TD, Daling JR. Characteristics of telephone survey respondents according to willingness to participate. Am J Epidemiol 2003; 157(1):6673.

36. Bowlin SJ, Morrill BD, Nafziger AN, Jenkins PL, Lewis C, Pearson TA. Validity of cardiovascular disease risk factors assessed by telephone survey: the Behavioral Risk Factor Survey. J Clin Epidemiol 1993; 46(6):561-71.

37. Costa NR, Pinto LF. Avaliação de programa de atenção à saúde: incentivo à oferta de atenção ambulatorial e a experiência da descentralização no Brasil. Rev C S Col 2002; 7(4):907-23.

38. Datasus. PNIIS - Política Nacional de Informação e Informática em Saúde. Brasília: MS; 2004.

39. Leão BF, Costa CG, Facchini LA, Bandarra EB, Goncalves SF, Bretas Jr N, et al. The Brazilian health informatics and information policy: building the consensus. Medinfo 2004; 11(Pt 2):1207-10.

40. Ribeiro JM, Costa NR, Pinto LFS, Silva PLB. Atenção ao pré-natal na percepção das usuárias do Sistema Único de Saúde: um estudo comparativo. Cad. Saúde Pública 2004; 20(2):534-45.

41. Donabedian A. Quality assurance. Structure, process and outcome. Nurs Stand 1992; 7(11 Suppl QA):4-5.

Artigo apresentado em 27/02/2006

Artigo aprovado em 31/03/2006

Versão final apresentada em 17/04/2006 